\title{
New Trends for Decision Support Systems
}

\author{
Hao-Tian Zhang, Student MIEEE, Qian Yang, Chun Sing Lai, Student MIEEE, Loi Lei Lai, FIEEE
}

\begin{abstract}
In this paper, Decision Support System (DSS) from 30 years ago to the near future will be studied to investigate the development trend of DSS. A typical DSS architecture has been described and the requirement of the DSS functionality implementation is illustrated in this article. Because all of the DSSs are projected-oriented, researchers are focused on developing general architecture to adapt as many cases as possible. Researchers begin to investigate the dynamic interaction and multi-criteria for the DSS application. In the near future, with the development of cloud computing, some cloud-based and agent-based DSSs will be adopted to various applications.
\end{abstract}

Keywords-Decision support system, cloud computing, computational intelligence

\section{INTRODUCTION}

$\mathrm{D}$ ecision Support System (DSS) is an information system, which is based on interactive computer to support decision making in planning, management, operations for power utility, business and organizations. The rapid changes on decision making, which cannot be easily identified, can be assisted by communication technologies and computer-based system compiling information gathered from a wide range of resources like raw data, documents, experts' experience \& knowledge, and business models [1]. Originally, decision support concept came from the theoretical studies of decision making for organizations by Carnegie Institute of Technology and technical practice on interactive computer systems by Massachusetts Institute of Technology in the 60s [1]. In 70s, 'decision support' began to grow among academia, and the first paper appeared in the conference and journal at that time. The DSSs have been introduced to China in 80s. In the earlier 90s, DSS applications are spread into different areas through data warehousing and on-line analytical processes. Since mid 90s, DSS starts to apply web-based analytical process [24]. Recently, with the development of cloud computing technology, DSS based on cloud computing technology has been proposed [21-23]. Throughout the development of DSS in the recent 30 years, there are more than 20 methods to implement the DSS. It is difficult to distinguish the best principle to solve decision making problems from others since proposed DSS systems are usually project-oriented. DSS has been involved in a plenty of technical areas which include

Hao-Tian Zhang is with State Grid Energy Research Institute, Beijing, China. He is now on leave from City University London, UK (Email: haotian.zhang.1@city.ac.uk)

Qian Yang and Loi Lei Lai are with State Grid Energy Research Institute, Beijing, China

Chun Sing Lai is with State Grid Energy Research Institute, Beijing, China. He is now on leave from Brunel University, London, UK transportation, electricity and resource dispatch and so on.

This paper overviews the development of DSS in the past 30 years, and proposes the future tend. The paper is organized as follows: Section II gives the framework requirement of the DSS, and typical architectures will be discussed. Section III is divided into three parts: past, present and future to look into the architectures for different purpose. Finally, a brief summary is made in the conclusion.

\section{REQUIREMENT FOR DECISION SUPPORT SYSTEM}

A DSS involves a number of scientific areas such as computer science, simulation technology, software programming and cognitive science and so on. Basically, there are three types of problems for decision making, namely structured, unstructured and semi-structured. Structured problems can be solved by standard solution techniques with clearly specified procedures to make a decision. Whereas the procedures of unstructured problems are unspecified in advance, and most of the decisions procedures are followed only once. In semi-structured problems, procedures for decision making can be specified but the optimal decision making cannot be verified. For different levels in organizations and business companies, the objectives of the DSSs are not the same. There are three different levels in companies and organizations. Firstly, strategic planning, including long term policies planning, is used for governing resource acquisition, utilization and disposition. Secondly, management control ensures the resources can be obtained and used effectively and efficiently to achieve the organization objectives. Finally, operation control ensures effective progress.

In general, the DSS is project-oriented and the functionality is always determined by system architecture. With different architectures, DSS functionalities can be summarized as follows:

1.Collecting, managing and providing the organization external information related to decision questions in domains like policy, economy, society, environment, market and technology.

2.Collecting, managing and providing the organization internal information related to decision questions in domains like order request, storage status, production capability and finance.

3. Collecting, managing and providing the feedback of each alternative decision execution such as contract processing, material supply plan, and production implementation.

4.Having a certain capability of data storage and managing mathematical models which are closely linked with 
decision making.

5.Having a certain capability of storing and providing frequently-used mathematical methodology and algorithm, such as regression analysis, linear programming, and computational intelligence models

6.Having a certain capability of easily adding and modifying data, model and algorithm.

7.Having a certain capability of flexibly processing, collecting, analyzing and forecasting data through models and methodology, thereby generating general message and projections.

8.Providing a friendly interface to communicate between man and machine, and functionality of graphic output. Also, there is a capability to meet the request of stochastic data query to answer some "what...if..." questions and so on.

9.Providing favorable functionality of data communication in order to ensure that the required data can be collected, processed and delivered to the user in time.

To include all the functionalities above, a typical DSS architecture should have an optimization toolbox, an online analytical processing toolbox, a data mining toolbox, a transaction processing system and a database as illustrated in Figure 1. Users can generate problems to the DSS and obtain the analyzed answers from the user interface. And a data mining toolbox can find the required data in large database where data related to the problem are located.

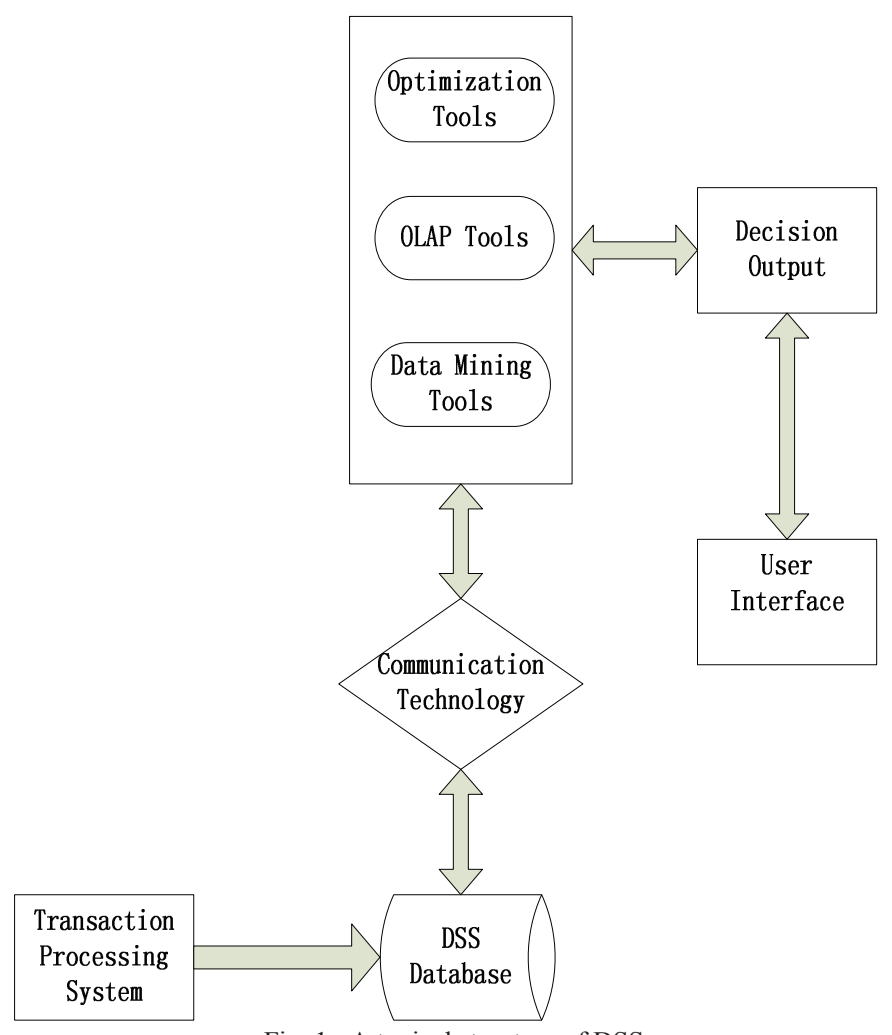

Fig. 1. A typical structure of DSS

\section{Requirements for DSS}

In general, there are five types of DSSs to achieve a decision making, namely, communication-driven DSS, data- driven DSS, document-driven DSS, knowledge-driven DSS and model-driven DSS [2]. No matter what kind of DSS is applied to a certain project, there are some generic requirements which are listed as follows:

1.Be compatible with as many decision processes and structures as possible.

2. Should have an interactive interface and friendly to users.

3. Can be accessed and controlled by users.

4. Allow end users to develop DSS without difficult.

5. Support modeling, data access and analysis.

6. Have an ability to work in both standalone and webbased environment.

Many areas have been involved with DSS. In reference [12], DSS for port planning was discussed. A Decision Support System for the Planning of the Workload on a grain terminal was illustrated in reference [13]. A DSS with an agent-based approach for oil refineries was demonstrated in reference [17]. References [23] and [22] gave examples of cloud-based DSS for traffic control and driver safety applications. In reference [14], the authors described a small helicopter landing case to present a DSS framework for dynamic multiple-criteria decision making.

\section{PASt, PRESENT AND FUtURE}

This section will give critical discussions about the development of Decision Support Systems. Also the promoting trend for future DSS system will be investigated.



Fig. 2. An XDSS approach [3]

\section{A. Past}

From 1970s to 1980s, comprehensive framework had been proposed to integrate with knowledge-based systems. The proposed DSS usually concentrates on the structure improvement during that time.

More domain-independent and user-friendly systems were 
developed by exploiting expert systems design in reference [3]. The proposed DSS structure as shown in Figure 2 was called XDSS. The knowledge base consisted of 5 areas, namely, domain, data dictionary, model, report generator and graphics knowledge. Each component in the architecture was maintained by experts. The domain knowledge would comprehend the issue that was defined by users and presented in the XDSS software. Then, the issue would be separated into sub-issues so that the relative knowledge base component could be adopted. Finally, a solution would come out through all sub-issues integrated with XDSS software.

A flexible logic-base DSS was proposed in reference [4]. The model developed by the authors adopts intentional database (IDB) for logic-based system. It means that the user could store a number of rules. However, there is another extensional database (EDB) for storing the actual data. Two kinds of models, namely, coded models and defined models, had been applied to the DSS system in order to reduce workload. The author believed that the DSS model achieved considerable development, and was used for design architectures based on IBM's top down approach so on.

Model management evolution was divided into 4 generations and has been described in [5]. Figure 3 demonstrated these 4 generations for DSS development. The author pointed out the advantages of the last generation models are:

1.The system could automatically integrate the model with data in order to reduce the programming redundancy.

2.Authorized user can access the model without many problems since many DSS systems were built in a distributed environment.

3.High compatibility enables the model management system to achieve more flexibility for DSS. Accommodating new database management system, model management system can reduce the impact of changing database models.

In reference [6], the framework of DSS for distributed computer system has been provided. Optional solutions were explored systematically by this methodology when objectives conflict with each other.

Three conceptual steps were developed to assess the DSS impact on organizational elements in reference [7]. The first step is to establish the DSS to identify the real consequence, at least in principle. The second step tries to recognize the subsets of the real consequence. And the last step is to evaluate both the recognized consequence and predicted consequence. The authors also discovered that the DSS could serve more when linking to an information network with all organizational centres connected together. Integrating with personal and organizational knowledge based systems is the trend pointed out by the authors.
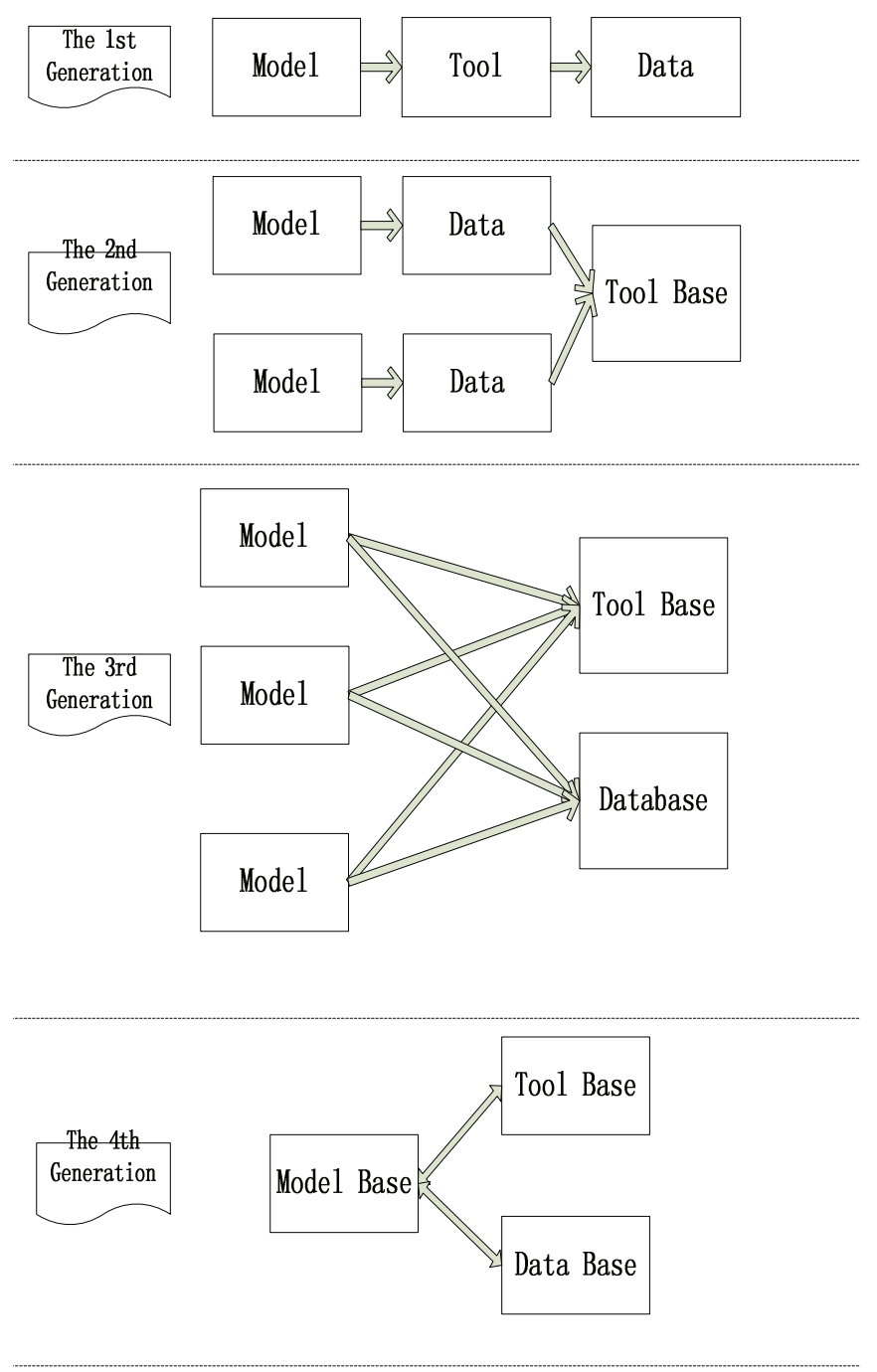

Fig. 3. 4 Generations for DSS development [5]

A framework of DSS for Computer-Integrated manufacturing has been discussed in reference [8]. In this survey, an information processing approach has been adopted to study the manufacturing environment. Some possible factors for designing the DSS system, such as the role of problem formulation and specification (involving goal setting), have been discussed in reference [9]. In 1985, a DSS system for global decision-making was reported. It summarized that the DSS would achieve more alternatives and better coordination [10]. Inquiry systems can be implemented for illstructured organizational problems and developing information systems. Strategies for creating a cooperative communication between expert system database and deduction have been outlined in reference [11]. There were many research papers debating on the appropriate mechanisms for coupling deduction and data component for an expert system. In reference [12], the authors proposed a new perspective in decision support system for port planning. A 2-layer information management system architecture was shown in Figure 4 to illustrate a conceptual distance from the logical to physical data structure. 


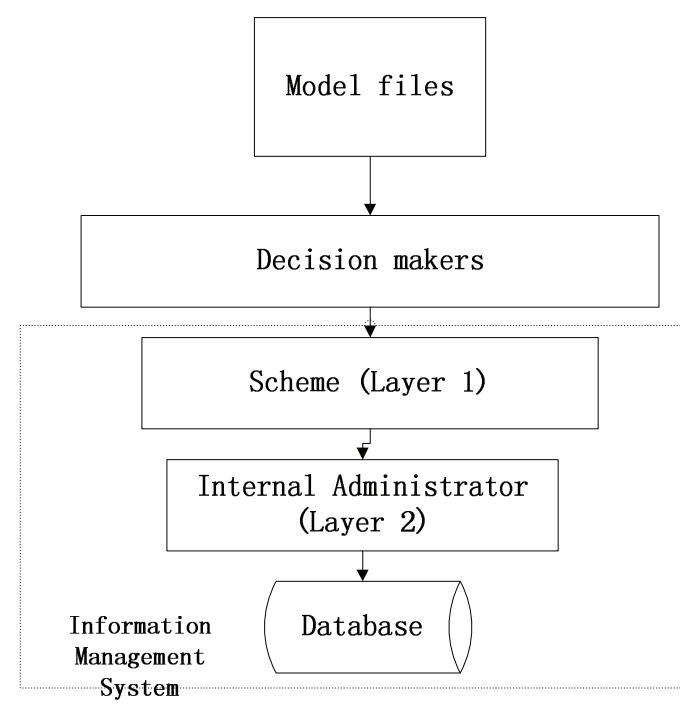

Fig. 4. DSS with 2-layer information management system [12]

\section{B. Present}

Around 2010s, DSS researchers began to pay attention to the multi-criteria decision making and dynamic interaction within a DSS. An increasing number of people realized that classical DSS cannot afford the dynamic changes in decision making of the real world. Besides, DSS frameworks proposed during that period also tried to minimize project risk.

Reference [14] proposed a new framework for dynamic multi-criteria decision support system. Classical multi-criteria decision making model would be used to find all available options. The proposed one introduced a dynamic environment where real world decision is taking place. It is worth noting that the versatile framework chooses a retention policy for historical options and can be widely used in many applications. For example helicopter landing was provided to illustrate the dynamic DSS system behavior.

In project management field there are uncertainties \& constraints, risks in projects and risk interaction need to be managed. A risk network model for decision support system in managing project risk was shown in Figure 5 [15]. The innovation of this framework is that the managerial suggestions can be modified, completed and refined, and also the managers can provide their knowledge to analyze the propagation behavior in the network.

A collaborative pattern mining for distributed information systems was described in reference [16]. Three frameworks have been compared with each other, and the advantage of the collaborative pattern (CLAP) has been listed. Self-contained mining framework is not efficient and feasible for fulfilling many objectives. CLAP was proposed to solve these problems by using communication network to exchange messages and database for information mining.

The authors in reference [17] added a dashboard between the decisions models and decision makers as illustrated in Figure 6 to consider both business and engineering decision variables, which are sometimes conflict with each other. To carry out sub-optimization for decision making, 2-stage decision based on optimization and agent-based models had been proposed. The first stage is to solve a multi-objective robust optimization problem by simulating integrated business and engineer models. The variables obtained in stage one would transfer to the next stage and process iterates until set criteria reached.

A measurement approach was derived to quantify dynamic interaction in knowledge based systems (KBS) based on control theory to examine the improvements [18]. The authors believed that the decision maker's initial attitude might affect the actual dynamic interaction.

Neural networks were proposed in reference [19] for decision making. To improve the decision making accuracy and reduce the misclassification, the authors have made comparisons among different cost index to investigate the best convergence. The final results made a clear picture that increasing number of cost index may lead to slower converging for related cost-sensitive decision.

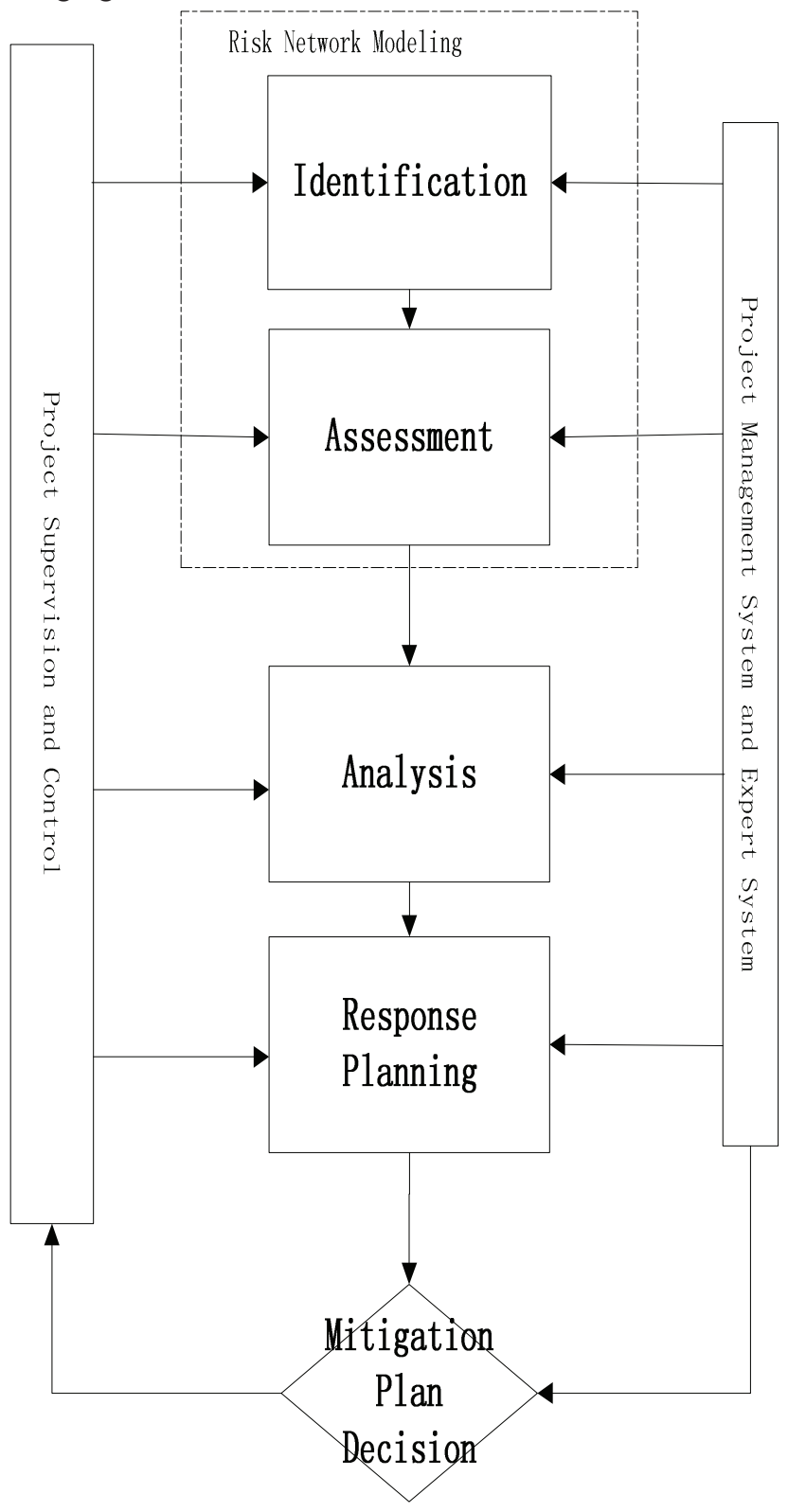

Fig. 5. DSS architecture for project risk management [15] 




Fig. 6. Business and engineering decision model with dashboard [17]

A text-based decision support system was proposed for financial sequence prediction in reference [20]. In this system, event sequences can be extracted from shallow text patterns and a classifier-based inference engine was applied for predicting the possibility of events occurring.

A shallow language model was provided to delete the incorrectly information. Also, the DSS system could verify the priority of event occurrence with both explicit and implicit knowledge participation during predicting. The other contribution was the inferece engine, which provided a robust and efficient means to predict financial texts. These contributions made the prediction accuracy of the model improved by $7 \%$ for un-seen data.



Fig. 7. DSS structure with cloud-computing technology

\begin{tabular}{|c|c|c|}
\hline Past & Present & Future \\
\hline $\begin{array}{l}\text { Concentrating on architecture } \\
\text { development: } \\
\text { 1. Applying XDSS framework } \\
\text { 2. Integrating model management } \\
\text { with data management } \\
\text { 3. Employing distributed computer } \\
\text { system } \\
\text { 4. Data inference for DSS } \\
\text { 5. Aggregating data } \\
\text { 6. Expert system for accessing } \\
\text { specific declaration } \\
\text { knowledge }\end{array}$ & $\begin{array}{l}\text { Concentrating on dynamic } \\
\text { interaction and multi-criteria } \\
\text { decisions: } \\
\text { 1. Risk network model for project } \\
\text { risk management } \\
\text { 2. Data mining methodology such } \\
\text { as SQLP, PALP and CLAP } \\
\text { 3. Agent-based approach } \\
\text { 4. Optimization methodology } \\
\text { 5. Textual information data }\end{array}$ & $\begin{array}{l}\text { Concentrating on data sharing and } \\
\text { information acquisition: } \\
\text { 1. Cloud computing technology } \\
\text { 2. Agent-based systems } \\
\text { 3. Security } \\
\text { 4. Integration with intelligent } \\
\text { methods, such as SVM, } \\
\text { swarm optimization and } \\
\text { evolutionary computing }\end{array}$ \\
\hline
\end{tabular}

Table. 1. DSS approaches and architectures from the past to the future

\section{Future}

Cloud computing was proposed for some future DSS systems for database and service sharing. Services like computation, data access, software applications and so on need to be delivered to the client. There are three service models comprising together to form a cloud computation environment, namely software as a service (SaaS), platform as a service (PaaS) and infrastructure as a service (IaaS). Figure 7 shows the DSS architecture with cloud computing technology.

A management system for traffic control applied agentbase was described in reference [22]. 4-layer framwork, namely, platform, application, unified and fabric layers have been proposed in cloud computing.

In reference [21], authors proposed a DSS architechture based upon cloud computing in order to meet the requirement of increasing demands of the information. Cloud is classified into three types: private cloud, public cloud and internal cloud. The aim of the cloud compution application in DSS is to reduce the complexity of managing the technology. Comparing with previous DSS systems, another benefit for applying cloud computing is that the demand can be scaled, the data centre can be streamlined, business processes can be improved and starting cost can be minimized. The authors in 
reference [23] demonstrated the requirements for cloud-based DSS system. 6 activities and proposed DSS framework and design clusters for traffic control has been described.

To implement DSS in a better way, communication technologies become more and more important to achieve data mining. As an important trend for communication development, cloud computing technologies will be involved for decision making. The past to the future trend has been discussed in Table 1.

\section{CONCLUSIONS}

This paper overviews the structure and projects in DSS development. The current survey gives a brief investigation in decision support system from the very beginning to the future. As a project-oriented structure, DSS cannot be compatible with every project. However, researchers devote themselves to make contributions to the DSS in order to design a general structure which can be applied into as many applications as possible. General DSS functionality and requirement have been discussed. Some classical DSS frameworks with graphical illustration have also been demonstrated. Cloud computing technology could make a real positive difference in the future, however, security could be a major issue and full attention should be given to this.

\section{REFERENCES}

[1] http://en.wikipedia.org/wiki/Decision_support_system, visited on 16 March 2012.

[2] Dr. Saeed Shiry, "Decision Support Systems", Introduction http://ceit.aut.ac.ir/ shiry/lecture/DSS/Introduction.ppt, visited on $3^{\text {rd }}$ of March, 2012

[3] Arun SEN and Gaytam Biswas, "Decision Support Systems: An Expert System Approach", Decision Support Systems 1, 1985, Elsevier Science Publisher B. V. (North-Holland), pp.197-204

[4] Michael C CHEN and Lawtence J. Henschen, "On the Use and Internal Structure of Logic-base DSS" Decision Support Systems 1, 1985, Elsevier Science Publisher B. V. (North-Holland), pp.205-219

[5] Ting-Peng Liang, "Integrating Model Management with Data Management in Decision Support Systems" Decision Support Systems 1, 1985, Elsevier Science Publisher B. V. (North-Holland), pp.221-232

[6] Amitava DUTTA and Hemant K. JAIN, “A DSS for Distributed Computer System Design in the presence of Multiple Conflicting Objectives" Decision Support Systems 1, 1985, Elsevier Science Publisher B. V. (North-Holland), pp.233-246

[7] H.K. Klein and R. Hirschheim, "Fundamental Issues of Decision Support Systems: A Consequentiality Perspective" Decision Support Systems 1, 1985, Elsevier Science Publisher B. V. (NorthHolland), pp.5-24

[8] Suranjan De, Shimon Y. Nof and Andrew B. Whinston, "Decision Support in computer-Integrated Manufacturing" Decision Support Systems 1, 1985, Elsevier Science Publisher B. V. (North-Holland), pp.37-56

[9] David Dery and Theodore J. Mock, "Information Support Systems for Problem Solving" Decision Support Systems 1, 1985, Elsevier Science Publisher B. V. (North-Holland), pp.103-109

[10] Henk G. SOL, "Aggregating Data for Decision Support" Decision Support Systems 1, 1985, Elsevier Science Publisher B. V. (North-Holland), pp.111-121

[11] Yannis Vassiliou, James Clifford and Mattias Jarke, “Access to Specific Declarative Knowledge by Expert System: The Impact of Logic Programming” Decision Support Systems 1, 1985, Elsevier Science Publisher B. V. (North-Holland), pp.123-141

[12] Hans-Herbert Wagschal, "New Perspective in Decision Support for Port Planning" Decision Support Systems 1, 1985, Elsevier Science Publisher B. V. (North-Holland), pp.277-292

[13] W.P.A van der Heyden and J.A. OTTJES, "A Decision Support System for the Planning of the Workload on a Grain Terminal" Decision Support Systems 1, 1985, Elsevier Science Publisher B. V. (North-Holland), pp.293-297

[14] Gianluca Campanella, Rita A. Ribeiro, "A framework for dynamic multiple-criteria decision making" Decision Support Systems 52, 2011, Elsevier Science Publisher B. V. (North-Holland), pp. $52-60$

[15] Chao Fang, Franck Marle, "A simulation-Based risk Network model for decision support in project risk management" Decision Support Systems 52, 2012, Elsevier Science Publisher B. V. (NorthHolland), pp.635-644

[16] Xingquan Zhu, Bin Li, Xindong Wu, Dan He, Chengqi Zhang, "CLAP: Collaborative pattern mining for distributed information systems" Decision Support Systems 52, 2011, Elsevier Science Publisher B. V. (North-Holland), pp.40-51

[17] W. Hu, A Almansoori, P.K. Kannan, S.Azarm, Z.Wang, "Corporate dashboards for integrated business and engineering decision in oil refineries: An agent-base approach: An agentbased approach" Decision Support Systems 52, 2012, Elsevier Science Publisher B. V. (North-Holland), pp.729-741

[18] Brandon A. Beemer, Dawn G Gregg, "Dynamic interaction in knowledge based systems: An exploratory investigation and empirical evaluation" Decision Support Systems 49, 2010, Elsevier Science Publisher B. V. (North-Holland), pp.386-395

[19] Guang-Zhi Ma, Enmin Song, Chih-Cheng Hung, Li Su, DongShan Huang, "Multiple costs based decision making with backpropagation neural networks" Decision Support Systems 52, 2012, Elsevier Science Publisher B. V. (North-Holland), pp.657-663

[20] Samuel W.K. Chan, James Franklin, "A Text-Based Decision Support System for Financial Sequence Prediction" Decision Support Systems 52, 2011, Elsevier Science Publisher B. V. (NorthHolland), pp.189-198

[21] Muzafar Ahmad Bhat, Razeef Mohd Shah, Bashir Ahmad, Inayat Rasool Bhat, "Cloud Computing: A Solution to Information Support Systems (ISS)" International Journal of Computer Applications, Vol. 11-No.5, Dec. 2010, pp 5-9

[22] ZhenJiang Li and Cheng Chen, Ka i Wang, "Cloud Computing for Agent-Based Urban Transportation Systems" Intelligent Transportation Systems, January/February 2011, 2011 IEEE, the IEEE Computer Society, pp73-79

[23] Shah Jahan Miah and Rakib Ahamed, "A Cloud-Based DSS Model for Driver Safety and Monitoring on Australian Roads", Int. J. Emerg. Sci., 1(4), Dec.2011, pp634-648

[24] http://dssresources.com/history/dsshistory.html, visited on 16 March 2012 